msh-mss Mathématiques et sciences humaines

193 | Printemps 2011

Varia

\title{
Structures d'ordres et sciences sociales
}

order structures and social sciences

\section{Bruno Leclerc et Bernard Monjardet}

\section{OpenEdition}

\section{Journals}

Édition électronique

URL : http://journals.openedition.org/msh/12004

DOI : $10.4000 / \mathrm{msh} .12004$

ISSN : $1950-682$

\section{Éditeur}

Centre d'analyse et de mathématique sociales de l'EHESS

\section{Édition imprimée}

Date de publication : 15 janvier 2011

Pagination : 77-97

ISBN : 09876936

ISSN : 0987-6936

\section{Référence électronique}

Bruno Leclerc et Bernard Monjardet, « Structures d'ordres et sciences sociales », Mathématiques et sciences humaines [En ligne], 193 | Printemps 2011, mis en ligne le 01 juin 2011, consulté le 23 juillet 2020. URL : http://journals.openedition.org/msh/12004; DOI : https://doi.org/10.4000/msh.12004

Ce document a été généré automatiquement le 23 juillet 2020.

(c) École des hautes études en sciences sociales 


\title{
Structures d'ordres et sciences sociales
}

\author{
Order structures and social sciences
}

Bruno Leclerc et Bernard Monjardet

\section{RÉSUMÉS}

On présente deux usages répandus de structures ordinales en sciences sociales. Le premier se rapporte à ce qui est souvent appelé l'analyse formelle des concepts, particulièrement utilisée en analyse et fouille des données. Le second concerne la " procédure médiane " présente aussi bien en théorie du choix social qu'en taxonomie mathématique. Aux origines de chacun de ces usages, on trouve des résultats innovants montrés par Marc Barbut, pour le premier à propos des échelles de Guttman de l'analyse hiérarchique et des treillis de Galois, pour le second, à propos des liens entre médiane et règle majoritaire de Condorcet.

We present two frequent uses of order structures in Social Sciences. The first one addresses what is often called Formal Concept Analysis, particularly used in data analysis and data mining. The second concerns the "median procedure" present in social choice as well as in mathematical taxonomy. At the origins of each of these uses, one finds innovative results due to Marc Barbut. In the first case, about Guttman scales in hierarchic analysis and about Galois lattices, in the second, about relations between medians and Condorcet's majority rule. 
INDEX

Mots-clés : analyse formelle des concepts, échelle de Guttman, majorité, médiane, ordre, treillis, treillis de Galois

Keywords : formal concepts analysis, Galois lattices, Guttman scale, lattice, majority, median, order

\section{AUTEURS}

BRUNO LECLERC

CAMS, EHESS, 190, avenue de France 75244 Paris cedex 13, leclerc@ehess.fr

BERNARD MONJARDET

CES, Université Paris 1-Panthéon-Sorbonne, MSE, 106-112, bd de l'Hôpital 75647 Paris cedex 13 et CAMS, EHESS, bernard.monjardet@univ-paris1.fr 